Research Article

\title{
Biomimetic Synthesis of Hydroxyapatite in Presence of Imidazole-4,5-dicarboxylic Acid Grafted Chitosan for Removing Chromium(VI)
}

\author{
Zhangxu Chen (D), Baorong Wu, Xiaodan Huang, Xianxue Li, and Yonglong Lin \\ Fujian Provincial Key Laboratory of Ecology-Toxicological Effects \& Control for Emerging Contaminants, \\ College of Environmental and Biological Engineering, Putian University, No. 1133, Xueyuan Road, Putian, \\ Fujian 351100, China \\ Correspondence should be addressed to Zhangxu Chen; xuzhangchen@163.com
}

Received 12 January 2018; Revised 30 March 2018; Accepted 11 April 2018; Published 3 June 2018

Academic Editor: Han Zhou

Copyright ( 12018 Zhangxu Chen et al. This is an open access article distributed under the Creative Commons Attribution License, which permits unrestricted use, distribution, and reproduction in any medium, provided the original work is properly cited.

In order to biomimetic synthesize hydroxyapatite similar to natural bone. Hydroxyapatite (HAP) is biomimetic synthesized in simulated body fluid (SBF) by addition of imidazole-4,5-dicarboxylic acid grafted chitosan (IDACS). The effect of molar ratio of chitosan (CS) to imidazole-4,5-dicarboxylic acid (IDA) on preparation of HAP was investigated. The structure, size, and crystal phase of the obtained hydroxyapatite were observed by Fourier transform infrared spectroscopy, X-ray powder diffraction, and scanning electron microscopy. The results show that the molar ratio of CS to IDA is $1: 3$, the temperature is $37.0^{\circ} \mathrm{C}$, the aging time is $48 \mathrm{~h}$, the synthesized nanorod-like hydroxyapatite with diameter $20-30 \mathrm{~nm}$, and length ranging from 75 to $120 \mathrm{~nm}$ presents excellent phase, which disperses well and is similar to the natural bone of HAP. The obtained HAP can be used to remove chromium(VI) by the orthogonal experiments, and the results indicated that the removal rate can reach $95.66 \%$ under the optimum conditions. These results suggest that the morphology of the obtained HAP is more affected by the material ratio of chitosan to imidazole-4,5-dicarboxylic acid than its structure, and the obtained HAP can effectively remove $\mathrm{Cr}(\mathrm{VI})$, which provides a novel method for biomimetic synthesis of other biomaterials and application in the water purification.

\section{Introduction}

Biomimetic synthesis of biological minerals has received considerable attentions in recent years. Calcium carbonate [1-3] and hydroxyapatite [4-6] are the most abundant biological minerals, which are especially focused all over the world. Up to date, synthesis of calcium carbonate and hydroxyapatite with various strategies has been studied. Among these, ethylene glycol [7] and chitosan [8] were used as organic matrix to biomimetic synthesize calcium carbonate and hydroxyapatite. Moreover, an impersonating physiological condition, simulated body liquid (SBF), has been generally utilized as a splashing medium since it was initially arranged by Kokubo et al. [9], which has specific preferences, such as, small particle sizes and narrow size dispersion. In recent years, biomimetic synthesis of hydroxyapatite was carried out in microwave oven using
$1.5 \times \mathrm{SBF}$ and $10 \times \mathrm{SBF}$ by Bindal et al. [10] and Tolga Demirtaş et al. [11]. It is worthwhile to mention that organic matrix is used as controlled template for biomimetic synthesis of calcium carbonate and hydroxyapatite in SBF solution.

Hydroxyapatite $\left(\mathrm{Ca}_{10}\left(\mathrm{PO}_{4}\right)_{6}(\mathrm{OH})_{2}\right.$; HAP $)$ is the one of the main biological materials, which can existed in human skeleton, animal bones, and teeth. Herein, the hydroxyapatite was formed through biomineralization and related biorelated processes, which are multifactorial, fantastic, and complex processes to commonly become a nice guide for fabrication of functional materials and keys for understanding of biophenomena [12-14].

HAP is used as a good biological material because it has particular structure and properties, such as compatibility, bioactivity, osteoconductivity, nontoxicity, and noninflammation. Therefore, it can be applied in the medical field and potentially 
water purification, such as an ideal implant material, pesticides, plastic surgery, protein adsorption, and removing heavy metal. As we known, lots of researchers have devoted themselves to the medical field. Unfortunately, it still represents great challenge to apply in water purification [15]. It is well known that heavy metal pollution in groundwater or wastewater is an important environment problem due to its toxic effects and accumulation throughout the food chain and hence in the human body [16].

Removing $\mathrm{Cr}(\mathrm{VI})$ is the most important and urgent problem to be solved as human body can absorb $\mathrm{Cr}$ (VI) easily through the digestive, respiratory, and mucous membrane [17]. During the last decade, numbers of technologies have been adopted to remove $\mathrm{Cr}(\mathrm{VI})$ from groundwater or wastewater. However, the available publications on removing $\mathrm{Cr}(\mathrm{VI})$ by hydroxyapatite and related composites are still very limited. HAP, HAP/chitin composite, and HAP/chitosan composite were synthesized to remove $\mathrm{Cr}(\mathrm{VI})$ by Kousalya et al., and the sorption capacities of them were found to be 2720,2845 , and $3450 \mathrm{mg} \cdot \mathrm{kg}^{-1}$, respectively [18]. Nanohydroxyapatite embedded gelatin composite was prepared by Gopalakannan, which was used as a biocompatible sorbent to the removal of hexavalent chromium from aqueous solution in batch mode. The adsorption capacity can reach $170 \mathrm{mg} \cdot \mathrm{g}^{-1}$, and the sorption of chromium(VI) onto Nanohydroxyapatite embedded gelatin biocomposite follows Langmuir isotherm [19].

In our previous research, a series of biological materials, such as calcium carbonate and hydroxyapatite have also been successfully fabricated by using the method of biomimetic synthesis $[8,20,21]$. Encouraged by this, herein, a rational design to biomimetic synthesize hydroxyapatite for removing $\mathrm{Cr}(\mathrm{VI})$ was firstly proposed. The obtained results might significantly contribute to the practical application of biological materials as promising composites in the treatment of contaminated water.

\section{Experimental Section}

2.1. Materials. Imidazole-4,5-dicarboxylic acid grafted chitosan (IDACS) is synthesized according to our previous reports [20-22]. Disodium hydrogen phosphate and $\left(\mathrm{Na}_{2} \mathrm{HPO}_{4}\right)$, absolute ethanol $\left(\mathrm{C}_{2} \mathrm{H}_{5} \mathrm{OH}\right)$, calcium chloride $\left(\mathrm{CaCl}_{2}\right)$, hydrochloric acid $(\mathrm{HCl})$, and ammonia $\left(\mathrm{NH}_{3} \cdot \mathrm{H}_{2} \mathrm{O}\right)$ are all of analytical reagent and are all purchased from Aladdin Reagent Co., Ltd. (China), which are used as starting materials without further purification.

\subsection{Biomimetic Synthesis of Hydroxyapatite in Presence of} IDACS. In this study, simulated body fluid (SBF) solution was used as solvent to replace water [23]. The hydroxyapatite crystals were obtained in pure water system [23]. To investigate the effect of IDACS on the crystallization of hydroxyapatite, different samples (S1-S5) were prepared by using IDACS as a matrix with the different molar ratio of chitosan to imidazole-4,5-dicarboxylic acid $(3: 1,2: 1,1: 1$, $1: 2$, and $1: 3$ ), respectively. In a typical synthesis, $0.050 \mathrm{~g}$ of different types of IDACS was added in $25.00 \mathrm{~mL}$ of $\mathrm{Na}_{2} \mathrm{HPO}_{4}$ $\left(0.06 \mathrm{~mol} \cdot \mathrm{L}^{-1}\right)$, which was adjusted to $\mathrm{pH} 10.0 \sim 10.5 \mathrm{using}$ ammonia or hydrochloric acid with constant stirring (Solution A). $\mathrm{CaCl}_{2}\left(0.10 \mathrm{~mol} \cdot \mathrm{L}^{-1}\right)$ was adjusted to $\mathrm{pH} 10.0 \sim 10.5$ using ammonia or hydrochloric acid with constant stirring (Solution B). Then, Solution B was dropwise added into the above Solution A kept at a temperature of $37^{\circ} \mathrm{C}$ and stirred for $30 \mathrm{~min}$ and aged for $48 \mathrm{~h}$. Finally, the obtained white precipitates were separated by centrifugation, rinsed several times with double distilled water and absolute ethanol, respectively, and then were dried in oven at $80^{\circ} \mathrm{C}$ until a constant weight was achieved. The as-synthesized product was denoted sample 1-5 (S1-5).

\subsection{Removing Cr(VI) by Obtained Nanorod-Like Hydroxyapa-} tite. In order to verify HAP be responsible for environmental remediation, its removing activities for chromium (VI) were investigated. The chromium sorption was studied as follows: $25 \mathrm{~mL}$ of chromium(VI) solution with various initial chromium(VI) concentrations were taken in a flask, and then the $\mathrm{pH}$ of the above solution can be adjusted by using $0.1 \mathrm{M} \mathrm{HCl} / \mathrm{NaOH}$ solution. After adjustment, different dosage of HAP5 was added into the above solution. To explore the optimum operating condition, the L16 $\left(4^{5}\right)$ orthogonal array was used in Table 1, herein, based on parallel single-factor experiment, the effect of $\mathrm{pH}$ value, initial concentrations of $\mathrm{Cr}(\mathrm{VI})$, dosage of HAP, and adsorption time on the removal of $\mathrm{Cr}(\mathrm{VI})$ were examined by our previous report [24].

2.4. Characterization. The sizes and morphologies of hydroxyapatite crystals were investigated by FESEM (Hitachi Model S-4800). Calibrated pellets of calcium carbonate (in proportion of $1 \mathrm{wt} \%$ in $\mathrm{KBr}$ powder) were performed and recorded with a Fourier transform infrared spectrometer (BRUKER TENSON 27, Germany) between 4000 and $400 \mathrm{~cm}^{-1}$ with a resolution of $4 \mathrm{~cm}^{-1}$. The X-ray diffraction (XRD) patterns were obtained on SmartLab $3 \mathrm{~kW}$, X-ray diffractometer (Rigaku, Japan) using $\mathrm{Cu} \mathrm{K} \alpha$ radiation at a scan rate of $4^{\circ} \mathrm{C} \cdot \mathrm{min}^{-1}$ was used to determine the identity of crystalline phase. The accelerating voltage and applied current were $30 \mathrm{kV}$ and $40 \mathrm{~mA}$, respectively.

The thermal behavior (TG) analysis of hydroxyapatite crystals were performed by using a Netzsch TG 209F3 in the temperature range of $40-1000^{\circ} \mathrm{C}$ with a heating rate of $10^{\circ} \mathrm{C} \cdot \mathrm{min}^{-1}$ in a dynamic atmosphere of nitrogen $\left(60 \mathrm{~mL} \cdot \mathrm{min}^{-1}\right)$. The surface area and the mean pore size were determined based on the nitrogen adsorption-desorption isotherms at liquid $\mathrm{N}_{2}$ temperature on a Micrometrics (ASAP 2020M + C) instrument. The specific surface area was determined by using the Brunauer-Emmett-Teller (BET) method. The mean pore size was estimated from the desorption branch of the nitrogen adsorption-desorption isotherms using the Barrett-Joyner-Halenda (BJH) method.

\section{Results and Discussion}

3.1. Influence of Molar Ratio of Chitosan/Imidazole4,5-dicarboxylic Acid on Hydroxyapatite. Figures 1-3 present the infrared spectra, XRD patterns, and FESEM images 
TABLE 1: Levels and factors of orthogonal experiment design.

\begin{tabular}{lcccc}
\hline Level & $\mathrm{pH}$ & $\begin{array}{c}\text { Initial } \\
\text { concentration } \\
\text { of } \mathrm{Cr}(\mathrm{VI})\left(\mathrm{mg} \cdot \mathrm{L}^{-1}\right)\end{array}$ & $\begin{array}{c}\text { Dosage of } \\
\mathrm{HAP} \\
\left(\mathrm{mg} \cdot \mathrm{L}^{-1}\right)\end{array}$ & $\begin{array}{c}\text { Adsorption time } \\
(\mathrm{min})\end{array}$ \\
\hline 1 & 3.00 & 25 & 200 & 90 \\
2 & 5.00 & 50 & 400 & 120 \\
3 & 7.00 & 100 & 800 & 150 \\
4 & 9.00 & 200 & 1000 & 180 \\
\hline
\end{tabular}

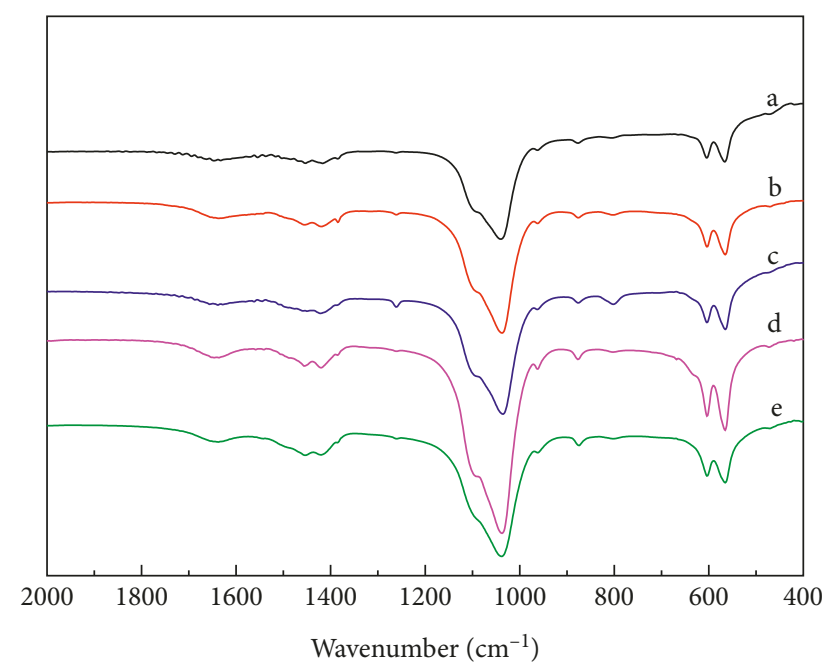

FIGURE 1: FT-IR spectra of HAP particles obtained by using IDACS as an organic matrix with different molar ratios of chitosan to imidazole-4,5-dicarboxylic acid. a: $3: 1 ;$ b: $2: 1 ;$ c: $1: 1$; d: $1: 2$; e: $1: 3$.

of the HAP crystals (S1-S5) produced with the different molar ratio of chitosan to imidazole-4,5-dicarboxylic acid $(3: 1,2: 1,1: 1,1: 2$, and $1: 3)$ at $37.0^{\circ} \mathrm{C}$ for aging $24 \mathrm{~h}$, respectively.

As it is well known, band assignment for FT-IR of HAP was well established [25]. These absorption bands corresponding to the infrared spectra of the synthesized powders are presented in Figure 1. The presence of two bands around 565 and $604 \mathrm{~cm}^{-1}$ correspond to characteristic $v_{4}(\mathrm{OPO})$ bending mode, and the $962 \mathrm{~cm}^{-1}$ band in the spectra was assigned to $\nu_{1}$ (PO) symmetric stretching. 1097 and $1038 \mathrm{~cm}^{-1}$ were assigned to $\nu_{3}(\mathrm{PO})$ antisymmetric stretching mode. The bands at around $1631 \mathrm{~cm}^{-1}$ can be indicated to the bending mode of $\mathrm{OH}^{-}$groups. It is worth noting that the weak peaks of 1462,1423 , and $872 \mathrm{~cm}^{-1}$ were observed in the five spectra, 1458 and $1419 \mathrm{~cm}^{-1}$ are corresponding to $v_{3}\left(\mathrm{CO}_{3}{ }^{2-}\right)$ antisymmetric stretching mode, and the band at $\sim 872 \mathrm{~cm}^{-1}$ are indicated to $v_{2}\left(\mathrm{CO}_{3}{ }^{2-}\right)$ bending mode, which is indicated that a small amount of carbonate hydroxyapatite was doped in HAP and they are similar to the main constituent of human bone [26], and it is different from HAP obtained in absence of imidazole-4,5dicarboxylic acid grafted chitosan [23].

The same observation can also be found by XRD analysis (S1-S5 in Figures 2(a)-2(e)). The diffraction peaks occurred at $2 \theta=25.8^{\circ}, 32.1^{\circ}, 33.9^{\circ}, 39.5^{\circ}, 46.8^{\circ}, 49.7^{\circ}$, and $53.5^{\circ}$, corresponding to HAP crystal face (002), (211), (202), (310),

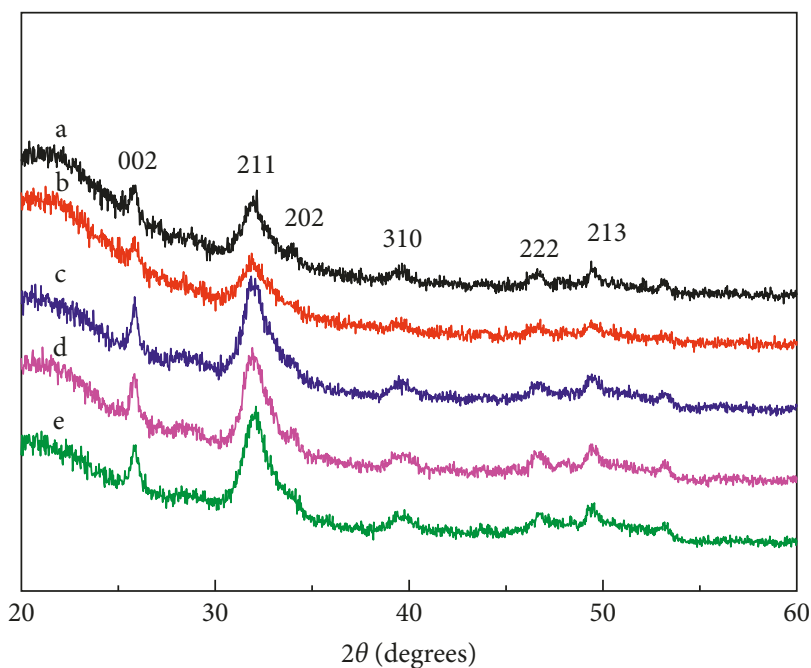

FIGURE 2: XRD patterns of HAP particles obtained by using IDACS as an organic matrix with different molar ratios of chitosan to imidazole-4,5-dicarboxylic acid. a: $3: 1 ;$ b: $2: 1 ;$ c: $1: 1$; d: $1: 2$; e: $1: 3$.

(222), (213), and (004), respectively (JCPDS number 03-0747) [19, 22]. It indicated that HAP was all formed with different molar ratio of chitosan to imidazole-4,5dicarboxylic acid $(3: 1,2: 1,1: 1,1: 2$, and $1: 3)$. Moreover, the intensity of X-ray diffraction patterns increased with the increasing the molar ratio of chitosan to imidazole4,5-dicarboxylic acid, especially in the face of crystal face (211).

In Figure 3, FESEM images of the HAP particles obtained at various molar ratio of chitosan to imidazole-4,5dicarboxylic acid show the morphology of HAP. When the molar ratio of chitosan to imidazole-4,5-dicarboxylic acid are $3: 1,2: 1,1: 1$, and $1: 2$, the obtained HAP presented aggregated spherical structure nanoparticles with mean diameters $\sim 30 \mathrm{~nm}$ (shown in Figures 3(a)-3(d)). As the molar ratio of chitosan to imidazole-4,5-dicarboxylic acid decreases to $1: 3$, nanorod-like HAP is with the welldistributed diameters of $20-30 \mathrm{~nm}$ and length ranging from 75 to $120 \mathrm{~nm}$. The above HAP with small particle sizes and narrow size dispersion can be biomimetic synthesized by using SBF solution. Based on the infrared spectra, XRD patterns, and FESEM images, it can be seen that the morphology of the obtained HAP is more affected by the material ratio of chitosan to imidazole-4,5-dicarboxylic acid than its structure.

The following reasons may explain these results, as the molar ratio of chitosan to imidazole-4,5-dicarboxylic acid decreases to $1: 3$, that is to say, using more imidazole-4,5dicarboxylic acid to modify chitosan, the synthesized imidazole-4,5-dicarboxylic acid grafted chitosan (IDACS), the positive charges on its surface are surplus because of the protonation of imidazole and carboxyl groups. Because of molecular recognition and electrostatic interactions, lots of $\mathrm{Ca}^{2+}$ ions will aggregate on the surface of IDACS and increase surface supersaturation of $\mathrm{Ca}^{2+}$ ions. Meanwhile, the bonding site is offered, which would attract $\mathrm{OH}^{-}$and $\mathrm{CO}_{3}{ }^{2-}$ 


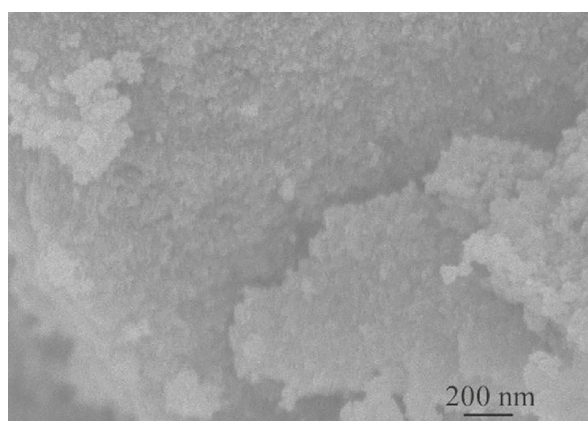

(a)

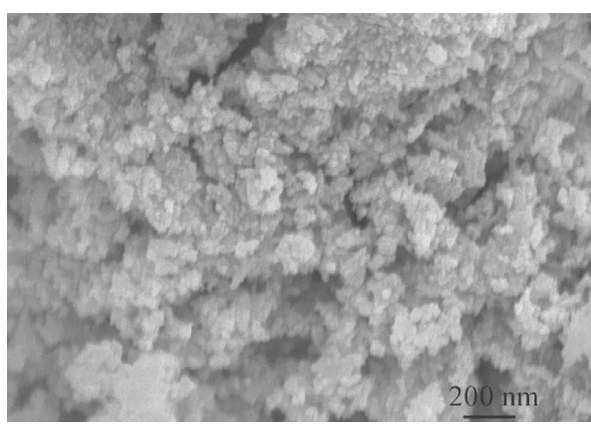

(c)

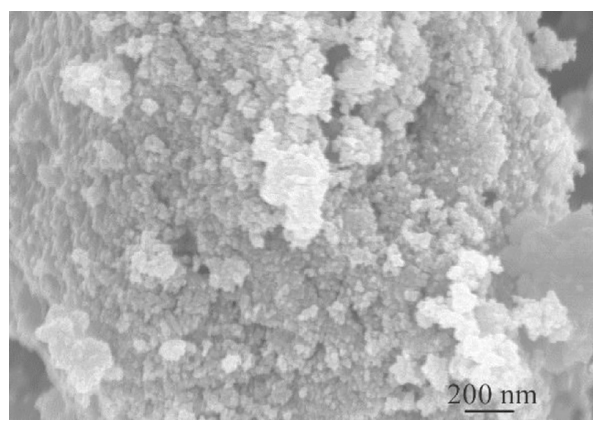

(b)

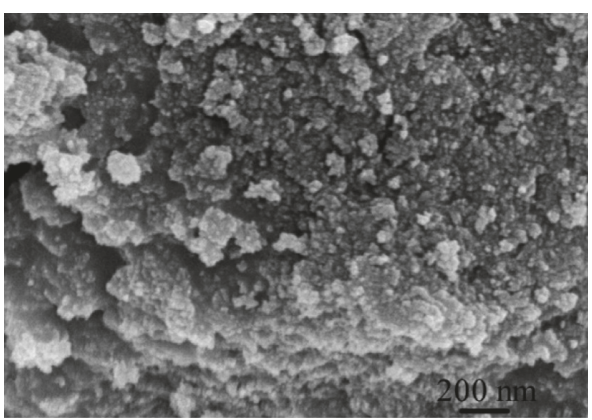

(d)

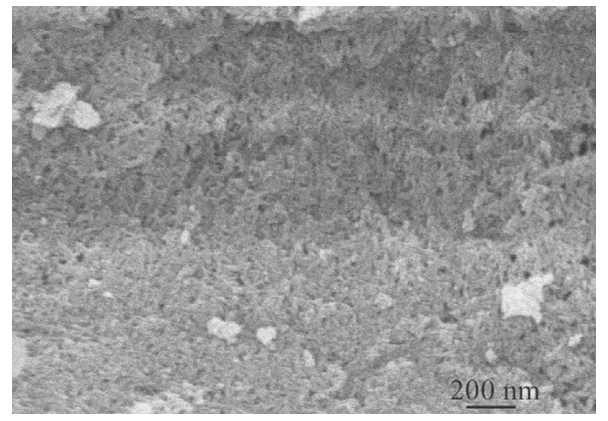

(e)

FIGURE 3: FESEM images of HAP particles obtained by using IDACS as an organic matrix with different molar ratios of chitosan to imidazole-4,5-dicarboxylic acid. (a) $3: 1$; (b) $2: 1$; (c) $1: 1$; (d) $1: 2$; (e) $1: 3$.

ions in the chain of IDACS and easily form the stable HAP nucleus and grow in the special crystal phase in special limited area.

3.2. Adsorption Studies. The value of the range shown in the orthogonal array indicated the influence of factors on the test index. Based on the intuitive analysis shown in Table 2, the range of $\mathrm{pH}$ value, initial concentration of $\mathrm{Cr}(\mathrm{VI})$, dosage of HAP, and adsorption time are 9.67, 4.76, 13.81, and 2.15 , respectively. It can be shown that the $\mathrm{pH}$ value and dosage of HAP are the most important key factors, while the others have insignificant effects. As seen on Table 2, the results revealed that the optimum degradation conditions are as follows: the range of $\mathrm{pH}$ value is 3.00 , initial concentration of $\mathrm{Cr}(\mathrm{VI})$ is $50 \mathrm{mg} \cdot \mathrm{L}^{-1}$, dosage of HAP is $1000 \mathrm{mg} \cdot \mathrm{L}^{-1}$, and adsorption time is $180 \mathrm{~min}$. Herein, the optimum degradation condition is different from the highest removal rate (number 4) in Table 2. Therefore, the removal rate need further research under the optimum degradation condition. Verifying test results show that the obtained HAP is feasible for removal of $\mathrm{Cr}(\mathrm{VI})$ under the optimum conditions, and the degradation rate can reach $95.66 \%$.

Based on the analysis of variance shown in Table 3, the dosage of HAP and $\mathrm{pH}$ value were significant influencing factors. The other factor is not significant factor. These results are agreement with the intuitive analysis.

3.3. Adsorption Mechanism. To understand the reason of removing chromium(VI) by HAP, the TG analysis, surface area, and porosity of the HAP materials are presented in Figures 4 and 5, respectively. The results obtained from the TG analysis are shown in Figure 4. As can be seen, the HAP5 obtained in SBF is stable, and the mass loss is about $2.4 \%$ of water (the weight loss below $100^{\circ} \mathrm{C}$ ), due to the removal of adsorbed water [27], and the weight loss in the temperature range $\left(40-900^{\circ} \mathrm{C}\right)$ investigated is $\sim 12.1 \%$. Furthermore, the 
TABLE 2: Orthogonal experimental design and results.

\begin{tabular}{|c|c|c|c|c|c|}
\hline Number & $\mathrm{pH}$ & $\begin{array}{c}\text { Initial } \\
\text { concentration of } \\
\mathrm{Cr}(\mathrm{VI})\left(\mathrm{mg} \cdot \mathrm{L}^{-1}\right) \\
\end{array}$ & $\begin{array}{c}\text { Dosage of } \\
\operatorname{HAP}\left(\mathrm{mg} \cdot \mathrm{L}^{-1}\right)\end{array}$ & $\begin{array}{l}\text { Adsorption } \\
\text { time (min) }\end{array}$ & $\begin{array}{c}\text { Removal } \\
\text { rate } \eta(\%)\end{array}$ \\
\hline 1 & 3 & 25 & 200 & 90 & 80.85 \\
\hline 2 & 3 & 50 & 400 & 120 & 86.32 \\
\hline 3 & 3 & 100 & 800 & 150 & 91.73 \\
\hline 4 & 3 & 200 & 1000 & 180 & 94.45 \\
\hline 5 & 5 & 25 & 400 & 150 & 82.05 \\
\hline 6 & 5 & 50 & 200 & 180 & 85.71 \\
\hline 7 & 5 & 100 & 1000 & 90 & 89.83 \\
\hline 8 & 5 & 200 & 800 & 120 & 88.36 \\
\hline 9 & 7 & 25 & 800 & 180 & 88.67 \\
\hline 10 & 7 & 50 & 1000 & 150 & 93.26 \\
\hline 11 & 7 & 100 & 200 & 120 & 73.95 \\
\hline 12 & 7 & 200 & 400 & 90 & 80.28 \\
\hline 13 & 9 & 25 & 1000 & 120 & 87.45 \\
\hline 14 & 9 & 50 & 800 & 90 & 83.62 \\
\hline 15 & 9 & 100 & 400 & 180 & 74.37 \\
\hline 16 & 9 & 200 & 200 & 150 & 69.25 \\
\hline Verifying test & 3 & 50 & 1000 & 180 & 95.66 \\
\hline ADR1* & 88.34 & 84.76 & 77.44 & 83.65 & \\
\hline $\mathrm{ADR} 2 *$ & 86.49 & 87.23 & 80.76 & 84.02 & \\
\hline ADR3* & 84.04 & 82.47 & 88.10 & 84.08 & \\
\hline $\mathrm{ADR}^{*}{ }^{*}$ & 78.67 & 83.09 & 91.25 & 85.80 & \\
\hline Range & 9.67 & 4.76 & 13.81 & 2.15 & \\
\hline $\mathrm{IF}^{*}$ & Secondary & Tertiary & Primary & General & \\
\hline
\end{tabular}

TAble 3: Analysis of variance.

\begin{tabular}{lccccc}
\hline Source of variation & SS & df & MS & F & Significance \\
\hline pH value & 211.18 & 3 & 70.39 & 10.20 & $*$ \\
Initial concentration of & 54.30 & 3 & 18.10 & 2.62 & \\
Cr(VI) & 489.07 & 3 & 163.02 & 23.63 & $*$ \\
Dosage of HAP & 11.12 & 3 & 3.71 & 0.54 & \\
Adsorption time & 20.70 & 3 & 6.90 & - & \\
Error & 786.37 & 15 & - & - & \\
Total &
\end{tabular}

F0.05 $(3,3)=9.28$; F0.01 $(3,3)=29.46 ;{ }^{*}$ a significant influencing factors.

isotherm adsorption curves for HAP5 are shown in Figure 5. In all cases, the isotherm curves have identical behaviors and can be classified as Type IV with loop H3, which is the typical characterization of mesoporous materials, suggesting the possibility of formation of slit-shape pores. Its surface area is $125.28 \mathrm{~m}^{2} \cdot \mathrm{g}^{-1}$, the larger surface area of HAP is, the better the adsorption is, which makes HAP5 possess good adsorption capacities for chromium(VI). The pore size distribution is centered in the range of $10-20 \mathrm{~nm}$, as shown in Figure 6 . The mean pore size during adsorption process was determined by the BET method. This value for HAP5 is $11.59 \mathrm{~nm}$, which is in agreement with the FESEM image of HAP5 in Figure 3(e).

\section{Conclusion}

Hydroxyapatite can be biomimetic synthesized successfully and fully characterized. The morphology of the obtained

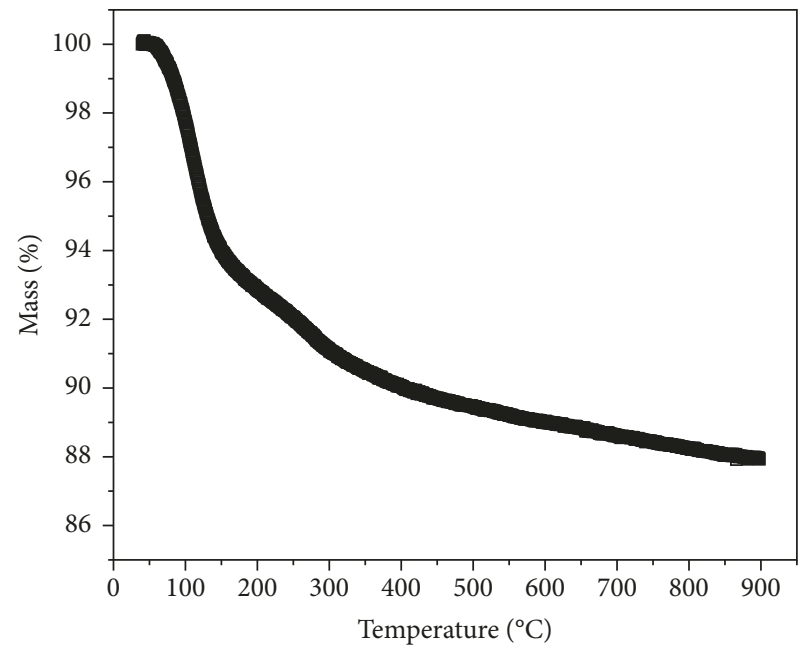

FIgURE 4: Thermal decomposition of HAP5.

HAP is more affected by the material ratio of chitosan to imidazole-4,5-dicarboxylic acid than its structure. The formed HAP can remove chromium(VI) effectively. It can be expected that the obtained biological materials might be used for removing heavy metal in the environmental remediation or in wastewater.

\section{Data Availability}

All data generated or analysed during this study are included in this published article. 


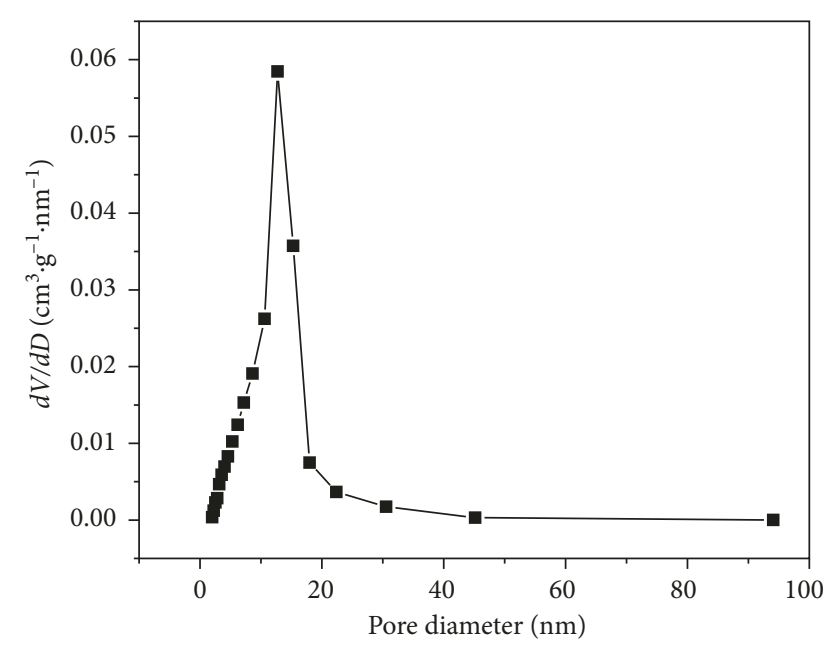

Figure 5: Pore diameter distributions of HAP5.

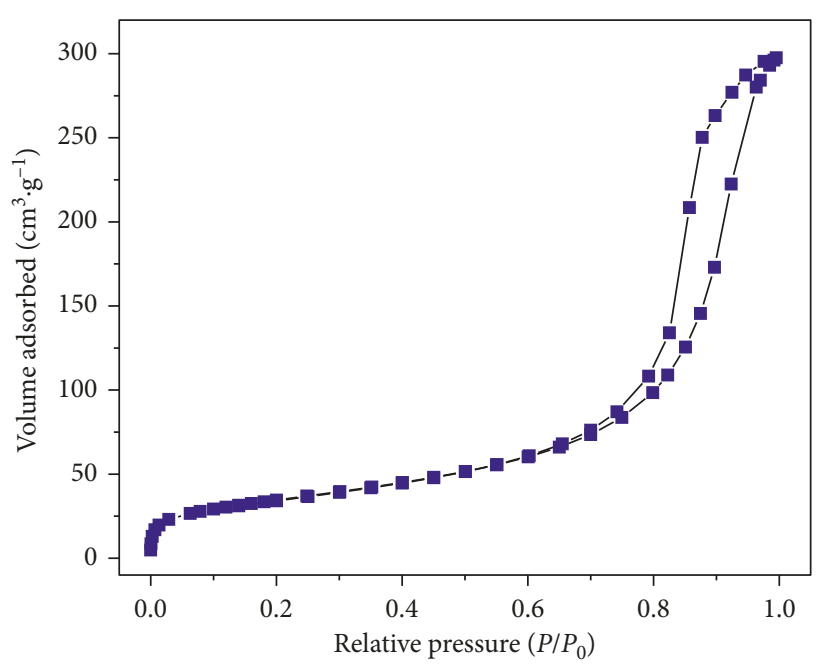

Figure 6: $\mathrm{N}_{2}$ adsorption-desorption isotherms of HAP5.

\section{Conflicts of Interest}

The authors declare that they have no conflicts of interest.

\section{Acknowledgments}

This work was supported by the National Science Foundation of China (51478449 and 51778598), Fujian Provincial Natural Science Foundation (2015J01644, 2017J01590, and 2017J01710), Scientific Research Plan of Education Bureau of Fujian Province (JAT160431), Projects of Putian University (2015060, 2016015, and 2016065), National College Students' Innovation and Entrepreneurship Training Program Project (201711498008, 201711498006 and 201811498011), Fujian College Students' Innovation, and Entrepreneurship Training Program Project (201711498037 and 201711498034).

\section{References}

[1] J. Wu and R. J. Zeng, "Biomimetic regulation of microbially induced calcium carbonate precipitation involving immobilization of sporasarcina pasteurii by sodium alginate," Crystal Growth and Design, vol. 17, no. 4, pp. 1854-1862, 2017.

[2] M. A. Lopez-Heredia, A. Łapa, A. C. Mendes et al., "Bioinspired, biomimetic, double-enzymatic mineralization of hydrogels for bone regeneration with calcium carbonate," Materials Letters, vol. 190, pp. 13-16, 2017.

[3] Z. Chen, B. Zheng, X. Huang et al., "Biomimetic synthesis of calcium carbonate by imidazole-1-acetic acid-grafted-chitosan," Journal of Functional Materials, vol. 48, no. 4, pp. 04085-04095, 2017.

[4] B. Li, L. Kan, X. Zhang et al., "Biomimetic bone-like hydroxyapatite by mineralization on supramolecular porous fiber networks," Langmuir, vol. 33, no. 34, pp. 8493-8502, 2017.

[5] A. Zaharia, V. Muşat, E. M. Anghel et al., "Biomimetic chitosan-hydroxyapatite hybrid biocoatings for enamel remineralization," Ceramics International, vol. 43, no. 14, pp. 11390-11402, 2017.

[6] F. Miculescu, A. C. Mocanu, G. E. Stan et al., "Influence of the modulated two-step synthesis of biogenic hydroxyapatite on biomimetic products' surface," Applied Surface Science, vol. 438, pp. 147-157, 2017.

[7] B. V. Parakhonskiy, Y. I. Svenskaya, A. M. Yashchenok et al., "Size controlled hydroxyapatite and calcium carbonate particles: Synthesis and their application as templates for SERS platform," Colloids and Surfaces B: Biointerfaces, vol. 118, pp. 243-248, 2014.

[8] X. Chen, M. Xin, M. Li, and Z. Chen, "Biomimetic synthesis of vaterite by $N$-succinyl-O-hydroxypropyl sulfonated chitosan," Chinese Journal of Materials Research, vol. 30, no. 1, pp. 31-37, 2016.

[9] T. Kokubo, H. Kushitani, S. Sakka, T. Kitsugi, and T. Yamamuro, "Solutions able to reproduce in vivo surfacestructure changes in bioactive glass-ceramic A-W3," Journal of Biomedical Materials Research, vol. 24, no. 6, pp. 721-734, 1990.

[10] S. Türk, I. Altınsoy, G. ÇelebiEfe, M. Ipek, M. Özacar, and C. Bindal, "Microwave-assisted biomimetic synthesis of hydroxyapatite using different sources of calcium," Materials Science and Engineering C, vol. 76, pp. 528-535, 2017.

[11] T. Tolga Demirtaş, G. Kaynak, and M. Gümüşderelioğlu, "Bone-like hydroxyapatite precipitated from $10 \times$ SBF-like solution by microwave irradiation," Materials Science and Engineering C, vol. 49, pp. 713-719, 2015.

[12] Z. Wang, P. Huang, O. Jacobson et al., "Biomineralizationinspired synthesis of copper sulfide-ferritin nanocages as cancer theranostics," ACS Nano, vol. 10, no. 3, pp. 3453-3460, 2016.

[13] M. Okaniwa, Y. Oaki, and H. Imai, "Morphology and orientation control of organic crystals in organic media through advanced biomimetic approach," Bulletin of the Chemical Society of Japan, vol. 88, no. 10, pp. 1459-1465, 2015.

[14] E. Firlar, T. Perez-Gonzalez, A. Olszewska, D. Faivre, and T. Prozorov, "Following iron speciation in the early stages of magnetite magnetosome biomineralization," Journal of Materials Research, vol. 31, no. 5, pp. 547-555, 2016.

[15] P. Tang, Y. Zhou, and Z. Xie, "Effects of hydroxyapatite addition on heavy metal volatility during tannery sludge incineration," Environmental Science and Pollution Research International, vol. 20, no. 7, pp. 4405-4413, 2013.

[16] H. B. Bradl, "Adsorption of heavy metal ions on soils and soils constituents," Journal of Colloid and Interface Science, vol. 277, no. 1, pp. 1-18, 2004.

[17] K. Zhang, H. Li, X. Xu, and H. Yu, "Synthesis of reduced graphene oxide/ $\mathrm{NiO}$ nanocomposites for the removal of 
$\mathrm{Cr}(\mathrm{VI})$ from aqueous water by adsorption," Microporous and Mesoporous Materials, vol. 255, pp. 7-14, 2018.

[18] G. N. Kousalya, M. R. Gandhi, and S. Meenakshi, "Removal of toxic $\mathrm{Cr}(\mathrm{VI})$ ions from aqueous solution using nanohydroxyapatite-based chitin and chitosan hybrid composites," Adsorption Science and Technology, vol. 28, no. 1, pp. 49-64, 2010.

[19] V. Gopalakannan and N. Viswanathan, "Development of nano-hydroxyapatite embedded gelatin biocomposite for effective Cr(VI) removal," Industrial and Engineering Chemistry Research, vol. 54, no. 50, pp. 12561-12569, 2015.

[20] Z. Chen, M. Xin, M. Li, J. Xu, X. Li, and X. Chen, "Biomimetic synthesis of coexistence of vaterite-calcite phases controlled by histidine-grafted-chitosan," Journal of Crystal Growth, vol. 404, pp. 107-115, 2014.

[21] Z. Chen, X. Li, and B. Zheng, "Can spherical vaterite be biomimetic synthesized by using histidine-grafted-chitosan as an organic matrix," Journal of Inorganic and Organometallic Polymers and Materials, vol. 27, no. 4, pp. 1014-1021, 2017.

[22] Z. Chen, M. Xin, and M. Li, "Preparation and characterization of histidine grafted chitosan porous scaffolds," Polymer Materials Science and Engineering, vol. 29, no. 9, pp. 152-156, 2013.

[23] Z. Chen, "Biomimetic synthesis of hydroxyapatite in presence of imidazole-1-acetic acid-grafted-chitosan scaffold," New Chemical Materials, vol. 45, no. 6, pp. 230-235, 2017.

[24] Z. X. Chen, B. Y. Zheng, M. L. Fu et al., "The sorption of chromium(VI) on nano-hydroxyapatite in simulated chromium(VI) wastewater," Journal of Shandong University of Technology, vol. 24, no. 5, pp. 20-22, 2010.

[25] Y. Deng, H. Wang, L. Zhang, Y. Li, and S. Wei, "In situ, synthesis and in vitro, biocompatibility of needle-like nanohydroxyapatite in agar-gelatin co-hydrogel," Materials Letters, vol. 104, pp. 8-12, 2013.

[26] J. J. Lovón-Quintana, J. K. Rodriguez-Guerrero, and P. G. Valença, "Carbonate hydroxyapatite as a catalyst for ethanol conversion to hydrocarbon fuels," Applied Catalysis A General, vol. 542, pp. 136-145, 2017.

[27] D. C. Manatunga, R. M. de Silva, K. M. Nalin de Silva, N. de Silva, and E. V. A. Premalal, "Metal and polymer-mediated synthesis of porous crystalline hydroxyapatite nanocomposites for environmental remediation," Royal Society Open Science, vol. 5, no. 1, pp. 171557-171571, 2018. 


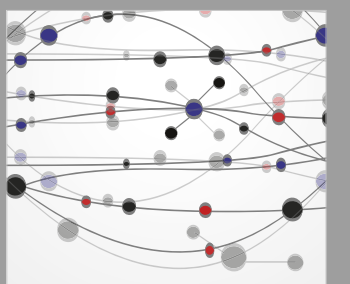

The Scientific World Journal
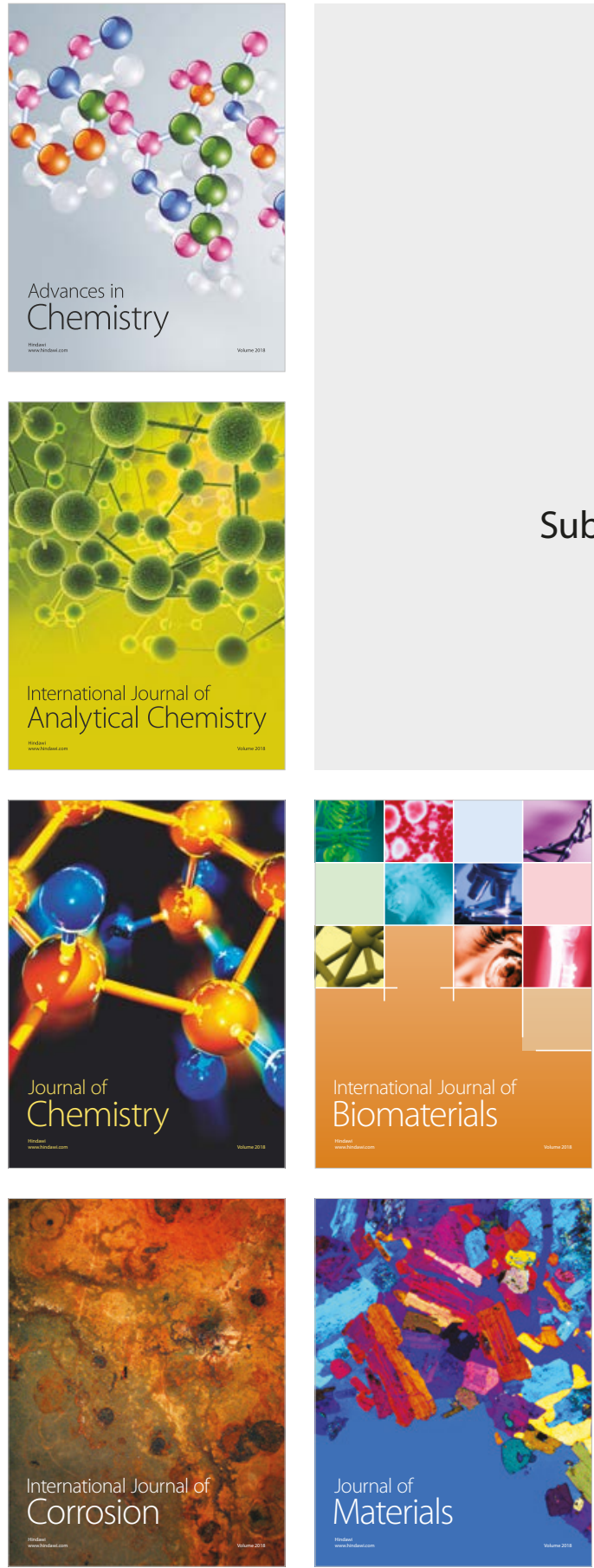

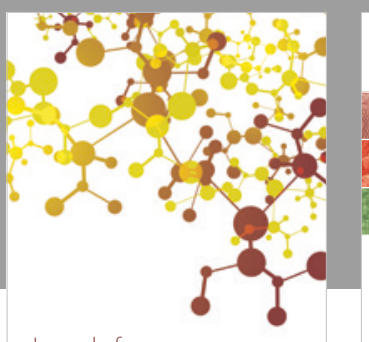

Journal of

Applied Chemistry
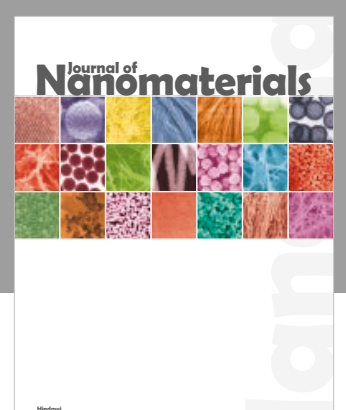

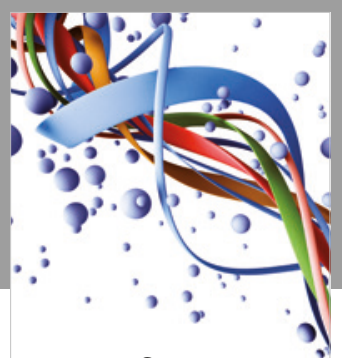

Scientifica

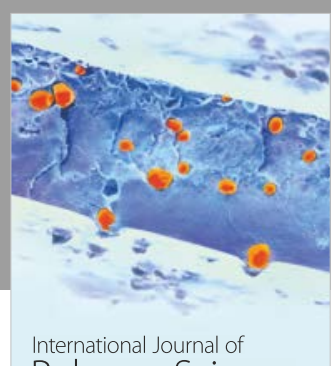

Polymer Science

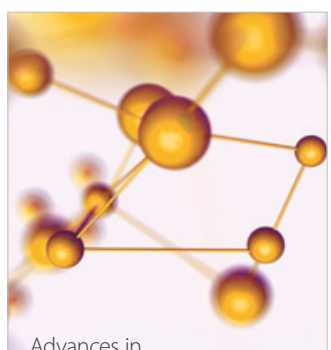

Physical Chemistry
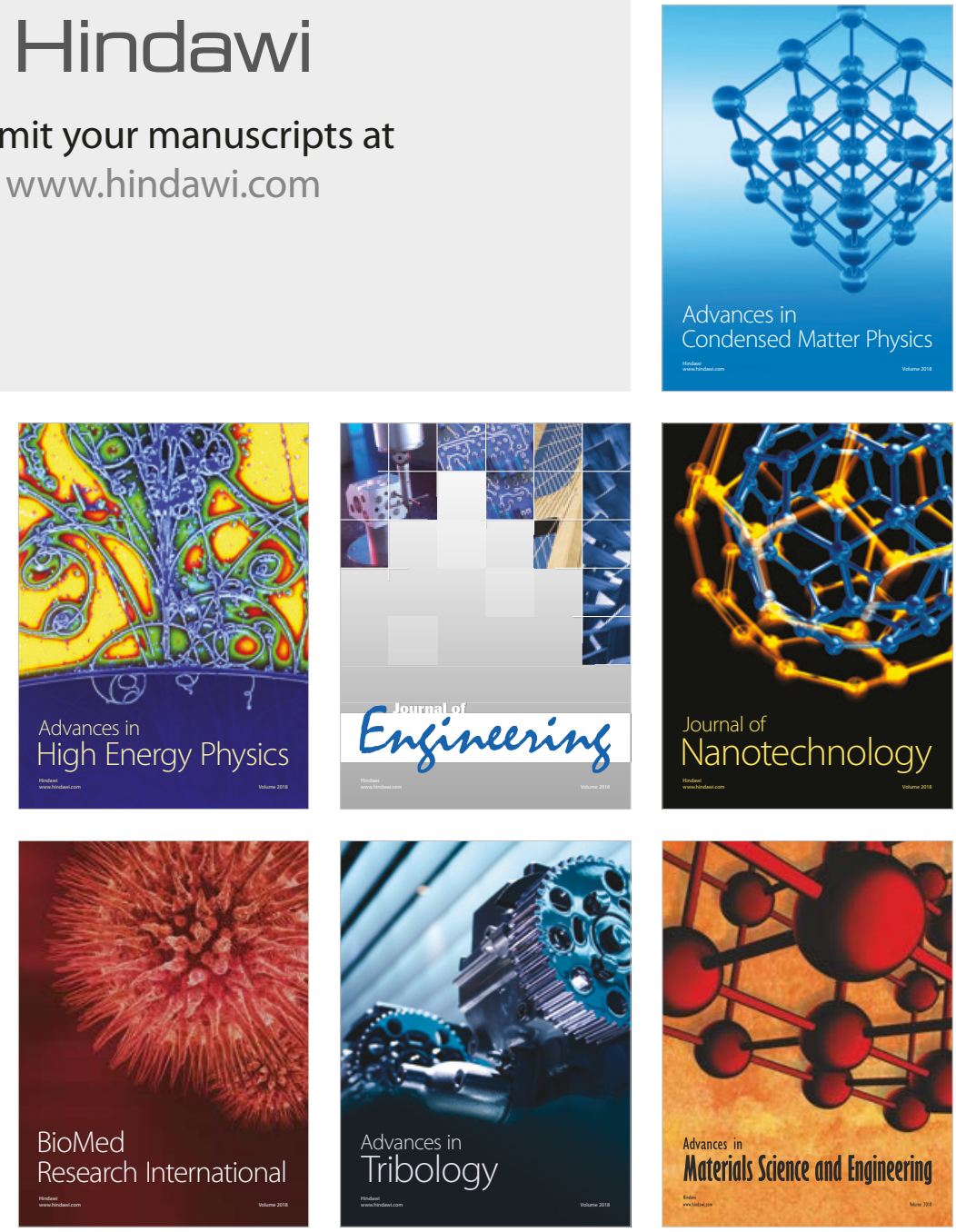